\title{
Nuclear Magnetic Resonance Spectroscopy for Medical and Dental Applications: A Comprehensive Review
}

\author{
Komal Zia ${ }^{1}$ Talal Siddiqui ${ }^{2}$ Saqib Ali $^{3} \quad$ Imran Faroo \\ ${ }^{1}$ Department of Oral Biology, Riyadh College of Dentistry and \\ Pharmacy, Riyadh, Saudi Arabia \\ ${ }^{2}$ General Dental Practitioner, Karachi, Pakistan \\ ${ }^{3}$ Department of Biomedical Dental Sciences, College of Dentistry, \\ Imam Abdulrahman Bin Faisal University, Dammam, Saudi Arabia \\ ${ }^{4}$ Department of Restorative Dentistry, College of Dentistry, Taibah \\ University, Madinah Munawwarah, Saudi Arabia \\ ${ }^{5}$ Department of Dental Materials, Islamic International Dental \\ College, Riphah International University, Islamabad, Pakistan \\ ${ }^{6}$ Department of Prosthodontics and Implantology, College of \\ Dentistry, King Faisal University, Al-Ahsa, Saudi Arabia
}

\author{
Address for correspondence Zohaib Khurshid, BDS, MRes, \\ MFGDP. Department of Prosthodontics and Implantology, \\ College of Dentistry, King Faisal University, Al-Ahsa, Saudi Arabia \\ (e-mail: drzohaibkhurshid@gmail.com).
}

Eur J Dent 2019;13:124-128

\begin{abstract}
Keywords

- dentistry

- metabolism

- multiple sclerosis

- spectroscopy

Nuclear magnetic resonance (NMR) spectroscopy is one of the most significant analytical techniques that has been developed in the past few decades. A broad range of biological and nonbiological applications ranging from an individual cell to organs and tissues has been investigated through NMR. Various aspects of this technique are still under research, and many functions of the NMR are still pending a better understanding and acknowledgment. Therefore, this review is aimed at providing a general overview of the main principles, types of this technique, and the advantages and disadvantages of NMR spectroscopy. In addition, an insight into the current uses of NMR in the field of medicine and dentistry and ongoing developments of NMR spectroscopy for future applications has been discussed.
\end{abstract}

\section{Introduction}

The electromagnetic spectra have been routinely used in the field of medicine and dentistry to detect abnormalities and fractures and to observe healing tissues, but this worthy detection tool comes with a risk of exposing the patients to excessive radiations. Although X-rays are swift and painless, long-term exposure to their radiations could cause harmful effects including cellular damage. Many new powerful analytical tools have been developed in recent years which can deliver precise results with minimal potential damage to the body tissues. ${ }^{1}$ Nuclear magnetic resonance (NMR) was first discovered in the 1940s. ${ }^{2}$ The NMR uses the magnetic properties of assured atomic nuclei and is widely being used in physics and chemistry. In dentistry, this technique is predominantly beneficial to explore the structure of amorphous glasses and dental cements, bioactive glasses interaction with oral tissues, identification of salivary metabolites for disease detection, ${ }^{3,4}$ and understanding the periodontal diseases by gingival crevicular fluid biomarkers analysis., ${ }^{5,6}$ It is also commonly used to review the fluoridation of apatite surfaces in the tooth structure. ${ }^{7}$ Therefore, this review is aimed at providing a general overview of the main principles of NMR, types of this technique, and the advantages and disadvantages of NMR spectroscopy. In addition, an insight into the current uses of NMR in the field of medicine and dentistry and ongoing developments of NMR spectroscopy for future applications is discussed.

\section{Basic Mechanism of Action of Nuclear Magnetic Resonance}

The basic principles of NMR are that the structural and chemical composition of different substances can be determined by their nuclei, which have their distinctive magnetic field. The basic NMR spectrometer analyzes using a magnetic field and a special detector to assess the changes ( $\boldsymbol{-}$ Fig. $\mathbf{1}$ ). The strength of the external magnetic field causes electrically 


\section{The NMR Spectrometer}

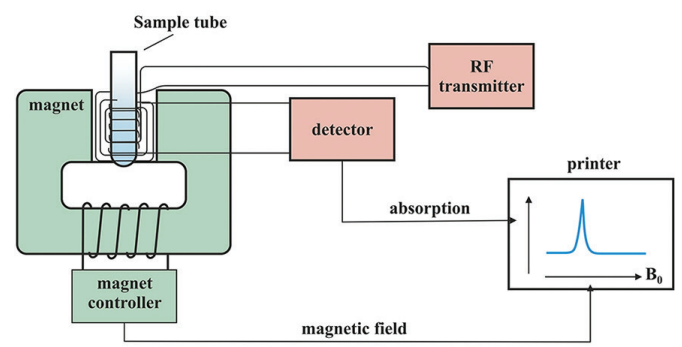

Fig. 1 Schematic presentation of a typical nuclear magnetic resonance spectrometer showing the relationship of various components (magnet, magnetic field, and detector).

charged nucleus to move from a lower energy level (E1) to a higher energy level (E2) and the difference between E2 and $\mathrm{E} 1$ is symbolized as $\Delta \mathrm{E}$ which is dependent on the power of the magnetic field and size of the nuclear field moment. ${ }^{8}$

The electromagnetic radiation rhythm attains the NMR signal with a frequency $(v)$ causing the nuclei to move to a higher energy level (E1/E2). When this electromagnetic radiation is stopped, it causes the nuclei to relax and accomplish thermal equilibrium. This release of energy from the nuclei is recorded in the form of spectra on the computer, and these spectra are exclusive for every nucleus and are equivalent to the energy levels between the two states (E2/E1) (

\section{Nuclear Spin}

The protons and neutrons of an atom exhibit spin. In some materials, the protons and neutrons exhibit paired spin such as carbon $12 \mathrm{C}$, oxygen 160 , and sulfur $32 \mathrm{~S}$, and in these cases, they cancel each other causing the nucleus not to spin. In some materials, the number of protons and neutrons in an atom are unpaired such as in proton ${ }^{1} \mathrm{H}$, phosphorus ${ }^{31} \mathrm{P}$, and fluoride ${ }^{19} \mathrm{~F}$.

\section{Applied Magnetic Field and the Nuclear Moment}

The spin of the protons and neutrons creates a magnetic moment in the nucleus of an atom. This magnetic moment could be toward the externally applied magnetic field or
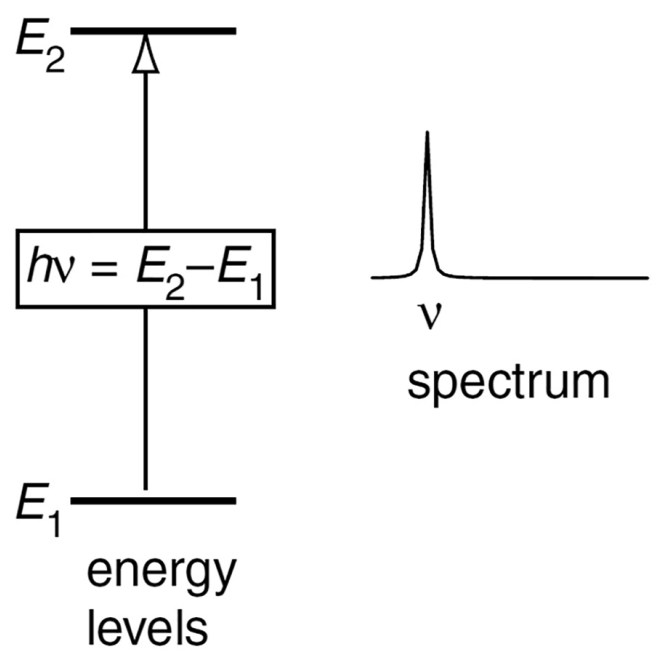

Fig. 2 A line in the spectrum is related to a transition between two energy levels (E2 and E1). ${ }^{9}$ away from it. B0 denotes this applied magnetic field. The more desired direction of the nuclear moment is toward the magnetic field which is at lower energy level denoted as $\alpha$ compared with the path opposite to the magnetic field denoted as $\beta^{5}$. The magnetic field is accountable for retaining the distinction in the energy levels. If it does not happen, all the orientations of the spin of $21+1$ would be of equal energy. ${ }^{2}$

\section{Magnetic Field Strength}

NMR requires a magnetic field that is both strong and uniform. The magnetic field strength is measured in Tesla or $\mathrm{MHz}$. The NMR requires a reference nucleus to represent the strength of the magnetic field.

\section{Chemical Shift}

The movement of the electrons creates a magnetic field in and around the nucleus. This magnetic field created is different in the direction as compared with the outer magnetic field. Any change in the magnetic field causes a similar change in the spectrum of the NMR. This sum of the shift is controlled by nature of the nucleus and nature of the motion of electrons in its surrounding atoms and molecules. This phenomenon is called "chemical shift (CS)." A reference compound is needed to measure $\mathrm{CS}^{9}$ and to determine and differentiate magnetically inequivalent nuclei present in a molecule.

\section{Spin-Spin Coupling}

The nuclei close to each other induce an incident called spinspin coupling (SS) due to the difference in nuclei's magnetic field direction. This direction could be either toward or opposing the magnetic field, causing the splitting of NMR signals. This magnetic field direction could either strengthen or fade the signals of NMR signals that can split into two or more components depending upon the specific nuclei having characteristic distance and relative potency. ${ }^{10}$

\section{Spin Relaxation (SR)}

Spin relaxation is the comeback of energy levels to stability. This occurs due to the loss of resonance signals with the passage of time after releasing the resonance frequency. There are two relaxation processes allowing the nuclear spin to return to stability, which are spin-lattice relaxation and spin-spin relaxation. ${ }^{11} T_{1}$ represents the time for a specific nucleus to come back to its thermal stability. Several the elements such as structure, molecular mass, temperature, and the solution could influence this procedure. ${ }^{12}$ SS relaxation happens when energy is lost from the nuclei/loss of signals. The energy lost is conveyed to nearby spin-active nuclei. SS relaxation time $\left(T_{2}\right)$ is the half-life of this procedure.

\section{Types of Nuclear Magnetic Resonance Spectroscopy}

Solid-state NMRs are used for chemical analysis to recognize any changes in the structure during phase transitions and different transformations in a solid state. The main technique frequently used in a solid-state NMR is magic angle spinning (MAS). This magic angle makes the resolution of the sample more precise by making the broader lines of the NMR 
narrower, ${ }^{13}$ resulting in narrower signals giving isotropic values and spinning sidebands to identify the CS of the nuclei for structural determination of solid materials.

\section{Phosphorus Nuclear Magnetic Resonance}

In the solid-state NMR, phosphorus is one of the isotopes used to study the molecules and structures of different samples. Compound classes of phosphorus were identified which included orthophosphate diesters, polyphosphate, phosphonates, orthophosphate monoesters, and orthophosphates. ${ }^{14}$

\section{Proton Nuclear Magnetic Resonance}

Proton is the initial and the most frequent atom to be used in NMR spectroscopy. It is also called hydrogen-NMR $\left({ }^{1} \mathrm{H}-\mathrm{NMR}\right)$ that provides information about the different varieties of hydrogen present in the molecule and also gives information about its adjacent surroundings. ${ }^{15}{ }^{1} \mathrm{H}$-NMR spectrum of main materials shows small CS range for usual compound is being studied. This CS ranges from +14 to $-14 \mathrm{ppm}$ and a broad difference in extent of coupling constant was observed. ${ }^{16}$

\section{${ }^{29}$ Silicon Magic Angle Spinning Nuclear Magnetic Resonance}

Silicon is an essential element, and its ${ }^{29} \mathrm{Si}$ isotope, which is used in ${ }^{29} \mathrm{Si}-\mathrm{NMR}$, has $4.70 \%$ natural occurrence with the half spin nucleus. It is another spectroscopic technique used to investigate the structures of organic compounds. Its value of the magnetic moment is a little low causing a low resonance frequency. The predominance of ${ }^{29} \mathrm{Si}-\mathrm{NMR}$ shifts is present in a range from +50 to -200 ppm. ${ }^{17}$

\section{${ }^{19}$ Fluorine Magic angle Spinning Nuclear Magnetic Resonance}

Isotopes of fluoride are naturally present in very less quantities except for the ${ }^{19} \mathrm{~F}$ isotope. $\mathrm{F}-19$ is the only constant isotope of fluorine found in large quantities. Due to its good nuclear qualities and a great quantity, it is used in ${ }^{19} \mathrm{~F}$ MAS NMR technique. ${ }^{19} \mathrm{~F}-\mathrm{NMR}$ technique is very rapid when compared with ${ }^{1} \mathrm{H}-\mathrm{NMR}$ technique, and without a doubt, ${ }^{19} \mathrm{~F}$ nucleus is one of the most amenable NMR nuclei. Fluorine has a spin of half-nucleus. Its nucleus in molecules is usually encircled by nine electrons, and its binding energy is $147,801 \mathrm{keV}$. The sensitivity of ${ }^{19} \mathrm{~F}-\mathrm{NMR}$ spectroscopy to its CS (to study the fine details of the local surrounding) is much high for fluorine, thus making it very reactive to NMR measurements with an extremely broad CS range. ${ }^{18}$ Yesinowski and Mobley ${ }^{19}$ verified the capacity of this NMR to differentiate between fluorapatite, fluorohydroxyapatite, and calcium fluoride in massive phase and also on hydroxyapatite surfaces. ${ }^{19} \mathrm{~F}-\mathrm{NMR}$ can identify fluoride even in minimal concentrations starting from $0.1 \% .{ }^{19} \mathrm{~F}-\mathrm{NMR}$ technique has also been used to study the metabolism of drugs containing fluoride. ${ }^{20}$

\section{${ }^{13}$ Carbon Nuclear Magnetic Resonance Spectroscopy}

This technique is a significant tool to recognize carbon atoms in any organic material. It also gives detailed information regarding the chemical structure of the organic compound being studied. ${ }^{13} \mathrm{C}$ is an isotope of carbon which has a spin quantum number of $1 / 2$ and is only $1.1 \%$ naturally present, and this isotope can be detected by ${ }^{13} \mathrm{C}$-NMR. ${ }^{13} \mathrm{C}$-NMR is less responsive to carbon in view of the fact that the main isotope of carbon is ${ }^{12} \mathrm{C}$, which is not magnetically active; therefore, it cannot be detected through this technique. The intensities of the signals in carbon-NMR are not usually comparative to the number of corresponding ${ }^{13} \mathrm{C}$ atoms. They are strongly reliant on the numerals of adjacent spins. ${ }^{21}$ Magnets utilized in C-NMR have a usual diameter of $10 \mathrm{~mm}$ and its usual range of $\mathrm{CS}$ is much larger compared with proton NMR. ${ }^{13} \mathrm{C}-\mathrm{NMR}$ can be used to find out the composition of different molecules and is also used in the drug industry to verify drug purity.

\section{${ }^{27}$ Aluminum Magic Angle Spinning Nuclear Magnetic Resonance}

${ }^{27}$ Aluminum MAS-NMR has a natural abundance of $100 \%$, and it has a 5/2 nuclear spin. The nucleus of aluminum is very responsive giving wide lines over the broad range of $\mathrm{CS}$. The main application of this NMR is to identify the existence of aluminum and to observe the probable structural changes of the different varieties of aluminum. In a previous study, ${ }^{27} \mathrm{Al}-\mathrm{NMR}$ has been used to observe alteration of $\mathrm{Al}$ (IV) into $\mathrm{Al}(\mathrm{VI})$ in the setting glass carbomer cement. ${ }^{22}$

\section{Advantages of Nuclear Magnetic Resonance Spectroscopy}

\section{Noninvasiveness}

The fundamental quality of NMR is its noninvasiveness. Due to NMR, the studies of biological cells and tissues are now possible without damaging the sample. The fact that both spectrum and imaging can be obtained without destroying the sample is noticeably the greatest advantage of NMR as in in vivo studies. ${ }^{23}$

\section{Lack of Ionizing Radiation}

Another major advantage of NMR is its lack of ionizing radiation. Many techniques are being used in vivo studies involving ionizing radiations. NMR has made it possible to avoid the exposure from radiation, which could be harmful to both the researcher and the subject. NMR utilizes isotopes which are stable such as carbon-13 to measure metabolic fluxes instead of radioactive compounds. NMR not only minimizes the exposure to observer and subject from the harmful rays but also eliminates the need to dispose of the radioactive tissues and other materials that might be contaminated during the study. ${ }^{1}$ Thus, the application of NMR can ensure the safety of the employees as well as reduce experimental cost due to the removal of discarded radioactive substances.

\section{Adjustability}

Extensive variety of processes can be investigated through NMR due to the flexibility of the particular technique that can be applied. This technique not only gives information about the physiology of the tissues, but it also gives great images of those tissues. With NMR, a single study can be performed with the same basic technique in both humans and animals, which is important to increase the translation of information. The computed tomography (CT) scan technique can only 
provide the imaging but not the metabolic or anatomic details, ${ }^{24}$ whereas NMR spectroscopy has the capability of acquiring a wide variety of information.

\section{Detailed Structural Analysis}

Over the period, NMR has played a major responsibility in determining the mechanisms and chemical connections at a molecular level. This technique has helped to obtain information regarding the minute details about the physical and chemical characteristics of structures. ${ }^{25}$ NMR can also analyze the parameters of CS, and it can give details on the local bonding environment around a particular atom, which could be calculated for the extended period of times with NMR. ${ }^{26}$ It utilizes the pseudo wave function to get information about large compound structures. ${ }^{27}$ NMR has the capability to assist studies of biochemical processes conducted in vivo, which is not efficiently achieved with other imaging techniques. Lee et $\mathrm{a}^{28}$ proposed that NMR is a better-quality technique as compared with X-ray diffraction in determining the archaeological bone structure.

\section{Disadvantages of Nuclear Magnetic Resonance Spectroscopy}

There are a few limitations.

\section{Presence of High Magnetic Field Surroundings Is Essential}

An unavoidable outcome when performing the NMR technique is the requirement to perform in a surrounding which has a high magnetic field. The presence of the magnetic field can affect the proper functioning of monitors and computer-controlled devices. If any sharp objects such as a scalpel, scissors, or stapler are present in the NMR magnetic field area, it can get attracted to the magnet field which can cause severe injuries to the workers. Nowadays, monitoring devices used in the magnetic field area are being designed to function properly under the magnetic field. ${ }^{29}$ Furthermore, instruments being used in NMR studies are made nonferromagnetic to reduce the problems encountered with high magnetic field surroundings. ${ }^{30} \mathrm{NMR}$ system cannot be purchased by a single investigator or for single research because of its high cost.

\section{Uses in Medicine and Dentistry}

CT scan images of the cranium are restricted by artifacts, but this limitation does not occur with NMR. In the field of medicine, NMR gives the benefit of identifying pediatric tumors, hematomas, and other pathologies. ${ }^{31}$ Since multiple sclerosis is a very tricky disease to identify, NMR has become the prime diagnostic device for multiple sclerosis. ${ }^{17}$ NMR has particular use for certain body areas such as brain where it produces very detailed and definite images showing delineation between gray and white matter, ${ }^{32}$ whereas some tissues such as bone, having low water percentage cannot emit strong signals to create images for NMR. ${ }^{33}$ Moreover, NMR is apparently victorious in identifying breast cancer at an early stage. According to a radiologist at Cleveland, a mammogram cannot differentiate between small cancer and a spot, when there are multiple cysts in the breast; however, with NMR, this distinction is possible. ${ }^{34}$ NMR technique also gives good images of fatty tissues, and a large quantity of fat creates wonderful images. In addition, the diagnosis of vascular diseases is promising with the $\mathrm{NMR}^{35}$ as it enables the detailed structural analysis of the surfaces of blood vessels and their abnormalities.

In dentistry, the aim of treatment is to preserve natural tissue and reconstruct the loss tissue with the help of biomaterials. These dental biomaterials are studied by many characterizing machines such as mechanical tester, physical testing, rheological testing, and biocompatibility testing. For that, NMR spectroscopy is a miracle machine to understand in-depth chemical reaction of materials ingredients and their effect with natural tissues. Extensive research on gas ionomer cement (GIC), resin composites, dental bone cements, and periodontal membranes materials has been conducted using the NMR spectroscopy. Prosser et al used NMR spectroscopy and reported the role of tartaric acid in the setting reaction of GIC, was "The fluid cement pastes have shown that tartaric acid reacts more readily than the polyacid with the glass, and hence suppresses the premature gelation of the cement." ${ }^{36}$

The cross-linking of $\mathrm{Al}$ in the setting of GIC is very crucial, and upon cement formation, $\mathrm{Al}$ ions in the glass are leached out from the surface layer of the glass, which is revealed by solid-state NMR spectroscopy. ${ }^{37}$ A novel antimicrobial polymeric dental restorative material was experimentally synthesized to see the biocompatibility, strength, and remineralization property by NMR $\left({ }^{1} \mathrm{H}-\right.$ and $\left.{ }^{13} \mathrm{C}-\mathrm{NMR}\right)$ spectroscopy. ${ }^{38}$ The advancements of proteomics in dentistry have brought a revolution in the management of oral diseases and analysis of molecular changes during the reconstruction or rehabilitation of oral tissues (soft and hard) with dental materials. ${ }^{39}$ To observe the orthodontically induced external apical root resorption biomarkers, Zhou et al studied the ${ }^{1} \mathrm{H}$-NMR-based metabolomics and detected the inflammatory metabolites from saliva samples. ${ }^{40}$ This study brings an importance of NMR spectroscopy in the field of clinical dentistry and dental early diagnosis. Another study on salivary metabolomics has reported the identification of several metabolic signatures from the control and sarcoidosis patients. ${ }^{41}$ The noninvasive, easy, and low-cost sampling of the human saliva attracted it as a diagnostic oral fluid, and by these omic devices such as NMR, more biomarkers can be explored. ${ }^{42}$

\section{Conclusions}

Considering the potential advantages of the NMR technique, it can be concluded with authority that it has become a preferred choice of technique for any diagnosis, treatment planning, maintenance of treatment and also to see the behavior of foreign materials interaction with the human body. NMR is still a growing technology, and it is being anticipated that few discoveries are now just around the corner.

\section{Financial Support and Sponsorship}

Nil. 


\section{Conflicts of Interest}

None declared.

\section{References}

1 Moseley I. Recent developments in imaging techniques. Br Med J (Clin Res Ed) 1982;284(6323):1141-1144

2 Grant DM, Harris RK. Encyclopedia of Nuclear Magnetic Resonance. Chichester, New York: John Wiley; 1996

3 Sahibzada HA, Khurshid Z, Khan RS, et al. Salivary IL-8, IL-6 and TNF- $\alpha$ as potential diagnostic biomarkers for oral cancer. Diagnostics (Basel) 2017;7(2):E21

4 Khurshid Z, Zafar MS, Khan RS, Najeeb S, Slowey PD, Rehman IU. Role of salivary biomarkers in oral cancer detection. Adv Clin Chem 2018;86:23-70

5 Zainuddin N, Karpukhina N, Law RV, Hill RG. Characterisation of a remineralising Glass Carbomer® ionomer cement by MAS-NMR spectroscopy. Dent Mater 2012;28(10):1051-1058

6 Khurshid Z, Mali M, Naseem M, Najeeb S, Zafar MS. Human gingival crevicular fluids (GCF) proteomics: an overview. Dent J (Basel) 2017;5(1) pii:E12.

7 Mohammed NR, Kent NW, Lynch RJ, Karpukhina N, Hill $\mathrm{R}$, Anderson P. Effects of fluoride on in vitro enamel demineralization analyzed by 19F MAS-NMR. Caries Res 2013;47(5):421-428

8 Keeler J. Understanding NMR Spectroscopy. Chichester: John Wiley \& Sons; 2011

9 Sørensen O, Eich G, Levitt MH, Bodenhausen G, Ernst R. Product operator formalism for the description of NMR pulse experiments. Prog Nucl Magn Reson Spectrosc 1984;16:163-192

10 Michal CA, Wehman JC, Jelinski LW. Deuterium quadrupole-coupling and chemical-shielding tensors in the model dipeptide glycylglycine monohydrochloride monohydrate. J Magn Reson B 1996;111(1):31-39

11 Hore P. Nuclear Magnetic Resonance. Oxford, UK: Oxford University Press; 2015

12 Akitt JW, Mann BE. NMR and Chemistry: An Introduction to Modern NMR Spectroscopy. 4th ed. London, UK: CRC Press; 2000

13 Robinson JN, Coy A, Dykstra R, Eccles CD, Hunter MW, Callaghan PT. Two-dimensional NMR spectroscopy in Earth's magnetic field. J Magn Reson 2006;182(2):343-347

14 Dubinnyi MA, Lesovoy DM, Dubovskii PV, Chupin VV, Arseniev AS. Modeling of 31P-NMR spectra of magnetically oriented phospholipid liposomes: a new analytical solution. Solid State Nucl Magn Reson 2006;29(4):305-311

15 Frydman L, Harwood JS. Isotropic spectra of half-integer quadrupolar spins from bidimensional magic-angle spinning NMR. J Am Chem Soc 1995; 117:5367-5368

16 Knight MJ, Webber AL, Pell AJ, et al. Fast resonance assignment and fold determination of human superoxide dismutase by highresolution proton-detected solid-state MAS NMR spectroscopy. Angew Chem Int Ed Engl 2011;50(49):11697-11701

17 Davis PL, Crooks LE, Margulis AR, Kaufman L. Nuclear magnetic resonance imaging: current capabilities. West J Med 1982;137(4):290-293

18 ten Cate JM. Review on fluoride, with special emphasis on calcium fluoride mechanisms in caries prevention. Eur J Oral Sci 1997;105(5 Pt 2):461-465

19 YesinowskiJP, Mobley MJ. Fluorine-19 MAS-NMR of fluoridated hydroxyapatite surfaces. J Am Chem Soc 1983;105:6191-6193

20 Koutcher JA, Sawyer RC, Kornblith AB, et al. In vivo monitoring of changes in 5-fluorouracil metabolism induced by methotrexate measured by 19F NMR spectroscopy. Magn Reson Med 1991;19(1):113-123
21 Fischer C, Lussi A, Hotz P. [The cariostatic mechanisms of action of fluorides. A review]. Schweiz Monatsschr Zahnmed 1995;105(3):311-317

22 Caytan E, Remaud GS, Tenailleau E, Akoka S. Precise and accurate quantitative (13)C NMR with reduced experimental time. Talanta 2007;71(3):1016-1021

23 Zainuddin N, Karpukhina N, Hill RG, Law RV. A long-term study on the setting reaction of glass ionomer cements by (27)Al MAS-NMR spectroscopy. Dent Mater 2009;25(3):290-295

24 Chang AE, Matory YL, Dwyer AJ, et al. Magnetic resonance imaging versus computed tomography in the evaluation of soft tissue tumors of the extremities. Ann Surg 1987;205(4):340-348

25 Hammer BE. Industrial applications of nuclear magnetic resonance. Sens Rev 1998;18:245-251

26 Duer Cambridge MJ. Introduction to Solid-State NMR Spectroscopy. Cambridge, UK: Wiley-Blackwell; 2005

27 Pickard CJ, Mauri F. All-electron magnetic response with pseudopotentials: NMR chemical shifts. Phys Rev B Condens Matter Mater Phys 2001;63:245101

28 Lee A, Klinowski J, Marseglia E. Application of nuclear magnetic resonance spectroscopy to bone diagenesis. J Archaeol Sci 1995;22:257-262

29 Damadian R, Minkoff L, Goldsmith M, Stanford M, Koutcher J. Field focusing nuclear magnetic resonance (FONAR): visualization of a tumor in a live animal. Science 1976;194 (4272):1430-1432

30 Bhujwalla ZM, Shungu DC, Chatham JC, Wehrle JP, Glickson JD. Glucose metabolism in RIF-1 tumors after reduction in blood flow: an in vivo $13 \mathrm{C}$ and 31P NMR study. Magn Reson Med 1994;32(3):303-309

31 Warren KE. NMR spectroscopy and pediatric brain tumors. Oncologist 2004;9(3):312-318

32 Ackerman JJ, Bore PJ, Gadian DG, Grove TH, Radda GK. N.m.r. studies of metabolism in perfused organs. Philos Trans R Soc Lond B Biol Sci 1980;289(1037):425-436

33 Anderson JH, Strandberg JD, Wong DF, et al. Multimodality correlative study of canine brain tumors. Proton magnetic resonance spectroscopy, positron emission tomography, and histology. Invest Radiol 1994;29(6):597-605

34 Bhakoo KK, Williams IT, Williams SR, Gadian DG, Noble MD. Proton nuclear magnetic resonance spectroscopy of primary cells derived from nervous tissue. J Neurochem 1996;66(3):1254-1263

35 Budinger TF. Image analysis in critical care medicine. Crit Care Med 1982;10(12):835-840

36 Prosser HJ, Richards CP, Wilson AD. NMR spectroscopy of dental materials. II. The role of tartaric acid in glass-ionomer cements. J Biomed Mater Res 1982;16(4):431-445

37 Pires RA, Nunes TG, Abrahams I, Hawkes GE. The role of aluminium and silicon in the setting chemistry of glass ionomer cements. J Mater Sci Mater Med 2008;19(4):1687-1692

38 Bienek DR, Frukhtbeyn SA, Giuseppetti AA, Okeke UC, Skrtic D. Antimicrobial monomers for polymeric dental restoratives: cytotoxicity and physicochemical properties. J Funct Biomater 2018;9(1):E20

39 Khurshid Z, Zohaib S, Najeeb S, Zafar MS, Rehman R, Rehman IU. Advances of proteomic sciences in dentistry. Int J Mol Sci 2016;17(5):E728

40 Zhou J, Hu H, Huang R. A pilot study of the metabolomic profiles of saliva from female orthodontic patients with external apical root resorption. Clin Chim Acta 2018;478:188-193

41 Duchemann B, Triba MN, Guez D, et al. Nuclear magnetic resonance spectroscopic analysis of salivary metabolome in sarcoidosis. Sarcoidosis Vasc Diffuse Lung Dis 2016; 33(1):10-16

42 Khurshid Z. Salivary point-of-care technology. Eur J Dent 2018;12(1):1-2 